\title{
Robust Control Analysis and Design for Discrete-time Singular Systems*†
}

\author{
CHUN-HSIUNG FANG, $\ddagger$ LI LEE $\S$ and FAN-REN CHANG $\|$
}

\begin{abstract}
A new approach to robust control analysis and design for uncertain discrete-time singular systems is proposed. Under the allowable structured perturbations, the stability robustness, regularity preservation, and impulse elimination are simultaneously guaranteed by the proposed method.
\end{abstract}

Key Words-Robust stability; robust control; structured perturbations; singular systems; regularity.

\begin{abstract}
In this paper, we propose a simple approach to analyse stability robustness of discrete-time singular systems under structured perturbations. The developed robustness criteria are then applied to solve robust regional poleassignment problems of singular systems. A robust control design algorithm, via state feedback, is also given. The robust stability problem of singular systems is more complicated than that of regular systems. Not only stability robustness but system regularity and impulse elimination should be considered simultaneously. Since the results of robust control and analysis for singular systems is not available in the literature as much as other fields, the paper may be viewed as a complementary result in this field. Although only discrete-time case is discussed, several results can be directly applied to continuous-time systems as well.
\end{abstract}

\section{NOTATION}

THE FOLLOWING notation will be used throughout the paper

$\rho(M)$ : spectral radius of $M \in \mathbb{C}^{n \times n}$

$|M|_{m}$ : modulus matrix of $M \in \mathbb{C}^{n \times n}$

$|M|:$ determinant of matrix $M \in \mathbb{C}^{n \times n}$

$|z|$ : magnitude of complex number $z$

$D(-h, f)$ : a disk centered at point $-h+j 0$ with radius $f$

\footnotetext{
* Received 4 August 1992; revised 10 August 1993; received in final form 10 February 1994. The original version of this paper was presented at the 12th IFAC World Congress which was held in Sydney, Australia, during 18-23 July 1993. The Published Proceedings of this IFAC Meeting may be oṛdered from: Elsevier Science Limited, The Boulevard, Langford Lane, Kidlington, Oxford OX5 1GB, U.K. This paper was recommended for publication in revised form by Associate Editor Vladimir Kurera under the direction of Editor Huibert Kwakernaak. Corresponding author Dr L. Lee. Fax 8867 5615137; E-mail leeli@ee.nsysu.edu.tw.

†This work was supported by the National Science Council of Taiwan, R.O.C. under Grant No. NSC-82-0404-E151-025.

$\ddagger$ Department of Electrical Engineering, National Kaohsiung Institute of Technology, Kaohsiung 807, Taiwan, R.O.C.

\& Department of Electrical Engineering, National Sun Yat-Sen University, Kaohsiung 804, Taiwan, R.O.C.

II Department of Electrical Engineering, National Taiwan University, Taipei 104, Taiwan, R.O.C.
}

$m_{i j}$ : the $(i, j)$ th element of matrix $M$

$M \geq N: \quad m_{i j}, n_{i j} \in \mathbb{R}$ and $m_{i j} \geq n_{i j}$ for $i, j=$ $1,2, \ldots, n$

$\Delta \Delta \Delta:$ end of statement in quoted lemmas Q.E.D.: end of proof.

\section{INTRODUCTION}

In the past ten years, there has been a growing interest in the system-theoretic problems of singular systems due to the extensive applications of singular systems to large-scale systems, circuits, economics, polynomial matrices and other areas (Luenberger, 1977; Verghese et al., 1981; Lewis, 1986; Dai, 1989; Fang and Chang, 1991, 1992). Sometimes the system is called generalized state-space systems, or implicit systems, or descriptor systems or semistate systems (Lewis, 1986). Several important and fundamental results in standard state-space systems have been successfully extended to singular systems (Cobb, 1984; Bender and Laub, 1987; Shayman, 1987; Zhou et al., 1987; Kucera and Zagalak, 1988; Fang and Chang, 1990). Many excellent design methods for singular system control have also been well developed recently (Kucera and Zagalak, 1991; Paraskevopoulos and Koumboulis, 1991). However, little effort has been devoted to studying the robust control problems of singular systems. In this paper, we will investigate such problems. Consider a discrete-time perturbed singular system

$$
E x(k+1)=A x(k)+\Delta A x(k), \quad E x(0)=E x_{0},
$$

where $E, A \in \mathbb{R}^{n \times n}$ and $\Delta A$ stands for the perturbations. Here the matrix $E$ may be singular. We will assume $\operatorname{rank} E \equiv r \leq n$. In 
system (1), $\Delta A$ denotes an $n \times n$ time-invariant structured perturbation matrix. The perturbations in the various elements of the system matrix $A$ are independent of one another (Sobel et al., 1989; Tesi and Vicino, 1990; Juang, 1991). In a practical situation, the perturbation matrix is not known exactly but the magnitude of the deviation which can be expected in the entries of $A$ may be known. The perturbation considered in this paper is described as

$$
|\Delta A|_{m} \leq q H
$$

where $q$ is a real positive number and $H$ is a constant nonnegative matrix. The constant matrix $H$ represents the highly structured information for the additive perturbation matrix $\Delta A$.

It has been known that a singular system generally contains three kinds of modes: dynamical finite modes, dynamical infinite modes, and nondynamical infinite modes (Verghese et al., 1981; Bender and Laub, 1987). The dynamical infinite modes can generate undesired impulsive behaviours. Hence, to eliminate or to avoid inducing dynamical infinite modes is a key work in singular system control (Wang et al., 1987). If we assume $\operatorname{deg}|z E-A|=r$ and $\Delta A=0$, the system (1) now has $r$ dynamical finite modes, no dynamical infinite modes, and $n-r$ nondynamical infinite modes. However, if the perturbation $\Delta A \neq 0$, it would possibly introduce dynamical infinite modes into system (1) since it can change the degree of $|z E-A-\Delta A|$. Furthermore, the perturbation $\triangle A$ can also possibly destroy the system regularity (i.e. $|z E-A-\Delta A|$ is identically zero). For example, let

$$
E=\left[\begin{array}{ll}
1 & 1 \\
0 & 0
\end{array}\right], \quad A=\left[\begin{array}{cc}
0.5 & 0.5 \\
4 & 4.2
\end{array}\right], \quad \Delta A=\left[\begin{array}{cc}
\epsilon & 0 \\
0.2 & 0
\end{array}\right]
$$

Here $\operatorname{deg}|z E-A|=1=\operatorname{rank} E$. It is easy to check $\operatorname{deg}|z E-A-\Delta A|=0<\operatorname{rank} E \forall \epsilon \neq 0$ and $|z E-A-\Delta A|$ becomes identically zero for $\epsilon=0$. This example indicates a fact that the robust control problems of singular systems must consider not only stability robustness but system regularity and impulse elimination. It should be noted that the latter two cases do not arise in the standard state-space systems. Therefore the robust control design problem of singular systems is more difficult than that of standard state-space systems.

This paper is organized as follows: Section 3 reviews some related results and states the problems concerned in this paper. The solutions to the problems are given in Section 4 . Section 5 makes a brief conclusion. Some examples are also provided at the end of each section for illustrating our ideas.

\section{RELATED LITERATURES AND PROBLEM STATEMENT}

\subsection{Review of related results}

In the literature, there are only a few papers dealing with the problems of robust control of singular systems. Mertzios (1984) developed a recursive formula to compute the approximated transfer function of perturbed singular systems. The sensitivity of poles and coefficients of transfer function to perturbations was also investigated. As the perturbations were given, Nichols (1986) used the condition number to measure the distance between nominal generalized eigenvalue and perturbed generalized eigenvalue. Based on the concept of eigenvalue sensitivity, Nichols proposed a nice algorithm to construct a state feedback gain such that the closed-loop system is insensitive to perturbations. However, since only first-order perturbation to eigenvalues is considered (other higher order perturbations are neglected), the perturbations allowed in her results must be small enough. Furthermore, her method can not be directly applied to calculate the upper bound of the perturbations under which the perturbed system remains stable. For checking the stability of perturbed systems, the perturbed ranges of all generalized eigenvalues must be computed. Syrmos and Lewis (1991) introduced a so-called chordal metric to replace the condition number used by Nichols and dealt with the problems in the same way as Nichols did. The problem of robust stability for singular systems with unstructured perturbations has been investigated by Qiu and Davison (1992). He and Ji (1992) proposed some sufficient conditions for practical stability of large scale systems with impulsive solutions. It seems that they are the first ones to discuss such a problem.

In this paper, from a different point of view, we propose a simple approach, which is an extension of Chou's (1990), to solve the robust control problem of singular systems. Since the matrix $E$ in this paper is not restricted to the identity matrix, Chou's results may be viewed as a special case of this paper. By our approach, it is easy to obtain an upper bound of perturbations under which the perturbed system is guaranteed regular, impulse-free and asymptotically stable. The restriction that perturbations must be small is removed. To check the stability of perturbed systems, it is not necessary to 
calculate the positions of all perturbed eigenvalues.

\subsection{Preliminaries and problem formulation}

To state our problem, we need to review some preliminary results.

Lemma 1. The system $\operatorname{Ex}(k+1)=A x(k)$ is said to be asymptotically stable if and only if (Lewis, 1986):

all roots of $|z E-A|=0$ lie inside

the disk $D(0,1)$.

$\Delta \Delta \Delta$

The statement of equation (3) can be replaced by

$$
|z E-A| \neq 0 \text { for all } z \text { with }|z| \geq 1 \text {. }
$$

Lemma 2. The following conditions are equivalent (Bender and Laub, 1987):

(a) the system $E x(k+1)=A x(k)$ has no dynamical infinite modes;

(b) $(z E-A)^{-1}$ is proper;

(c) $\operatorname{deg}|z E-A|=\operatorname{rank} E$.

$\Delta \Delta \Delta$

Note that it is not necessary to consider the dynamical infinite modes in standard state-space systems $(E=I)$ since condition (5b) is always true for such system.

Definition 1. For any two $n \times n$ real constant matrices $E$ and $A$, the pair $(E, A)$ is said to be regular, impulse-free (i.e. without dynamical infinite modes), and asymptotically stable if $|z E-A| \equiv 0,(z E-A)^{-1}$ is proper, and all roots of $|z E-A|=0$ lie within the disk $D(0,1)$.

Three robust control problems are investigated in this paper. (1) Assume the pair $(E, A)$ is regular, impulse-free, and asymptotically stable. What is the upper bound of perturbation $\Delta A$ such that the perturbed system (1) is still regular, impulse-free and asymptotically stable? (2) If the roots of $|s E-A|=0$ lie inside a specified disk, what is the upper bound of $\Delta A$ such that the roots of $|s E-A-\Delta A|=0$ still lie inside the disk? This is called the robust root-clustering problem (Yedavalli, 1993). (3) How to design a robust controller for the perturbed system $E x(k+1)=(A+\Delta A) x(k)+(B+\Delta B) u(k)$ by using the results developed in problems (1) and (2) is the third problem. In the above, $u(k)$ is the input signal and $\Delta A$ and $\Delta B$ represent the uncertainties on matrices $A$ and $B$, respectively.
4. SOLUTIONS

\subsection{Robust stability analysis}

Lemma 3. For any $n \times n$ constant matrices $X, Y, Z$ with $|X|_{m} \leq Z$, it is easy to obtain the following inequalities (Lancaster and Tismenetsky, 1985):

(a) $|X Y|_{m} \leq|X|_{m}|Y|_{m} \leq Z|Y|_{m}$

(b) $|X+Y|_{m} \leq|X|_{m}+|Y|_{m} \leq Z+|Y|_{m}$

(c) $\rho(X) \leq \rho\left(|X|_{m}\right) \leq \rho(Z)$

(d) $\rho(X Y) \leq \rho\left(|X|_{m}|Y|_{m}\right) \leq \rho\left(Z|Y|_{m}\right)$

(e) $\rho(X+Y) \leq \rho\left(|X+Y|_{m}\right) \leq \rho\left(|X|_{m}+|Y|_{m}\right)$

$$
\leq \rho\left(Z+|Y|_{m}\right) \text {. }
$$

The pair $(E, A)$ in perturbed system (1) is assumed regular, impulse-free, and asymptotically stable. We define $G_{p}(z) \equiv(z E-A)^{-1}$ and denote by $G(k)$ the pulse response sequence matrix of the multivariable system $G_{p}(z)$. One can rewrite $G_{p}(z)$ as

$$
G_{p}(z)=(z E-A)^{-1}=\sum_{k=0}^{\infty} G(k) z^{-k} .
$$

Then we have the following key lemma.

Lemma 4. If the pair $(E, A)$ is regular, asymptotically stable and impulse-free, and $|\Delta A|_{m} \leq q H$, then

$$
\begin{aligned}
\rho\left((z E-A)^{-1} \Delta A\right) \leq & \rho(q T H), \\
& \text { for all } z \text { with }|z| \geq 1
\end{aligned}
$$

where $T$ is defined by

$$
T \equiv \sum_{k=0}^{\infty}|G(k)|_{m}
$$

Proof. From Lemma 3 and equation (9), it is easy to check

$$
\begin{aligned}
& \rho\left((z E-A)^{-1} \Delta A\right. \\
& \quad \leq \rho\left(\left|(z E-A)^{-1} \Delta A\right|_{m}\right) \text { for all } z \text { with }|z| \geq 1 \\
& \quad \leq \rho\left(\left|\sum_{k=0}^{\infty} G(k) z^{-k}\right|_{m} \cdot|\Delta A|_{m}\right) \\
& \quad \leq \rho\left(\sum_{k=0}^{\infty}\left|G(k) z^{-k}\right|_{m} \cdot q H\right) \text { for all } z \text { with }|z| \geq 1 \\
& \quad \leq \rho\left(q \sum_{k=0}^{\infty}|G(k)|_{m} H\right) \text { with }|z| \geq 1 \\
& \quad=\rho(q T H) .
\end{aligned}
$$


The matrix $T$ in equation (11) would be finite if the pair $(E, A)$ is asymptotically stable and impulse-free. This fact can be verified in Remark 1 below.

Remark 1. By the Weierstrass decomposition (Dai, 1989; Lewis, 1986), we propose a simple method to evaluate the matrix $T$ without performing the expansion of $(z E-A)^{-1}$ required in equation (9). Since the pair $(E, A)$ is asymptotically stable and impulse-free, it can be transformed to the well-known Weierstrass form, i.e. there exist two constant nonsingular matrices $U$ and $V$ such that

$$
U(z E-A) V=\left[\begin{array}{cc}
z I_{r}-A_{r} & 0 \\
0 & I_{n-r}
\end{array}\right],
$$

where $A_{r} \in \mathbb{R}^{r \times r}$ and all eigenvalues of $A_{r}$ are within $D(0,1)$. Suppose $U$ and $V$ are decomposed as

$$
U=\left[\begin{array}{l}
U_{a} \\
U_{b}
\end{array}\right] \text { and } V=\left[\begin{array}{ll}
V_{a} & V_{b}
\end{array}\right]
$$

where $U_{a} \in \mathbb{R}^{r \times n}, \quad U_{b} \in \mathbb{R}^{(n-r) \times r}, \quad V_{a} \in \mathbb{R}^{n \times r}$ and $V_{b} \in \mathbb{R}^{n \times(n-r)}$. Then it is easy to show that

$$
\begin{aligned}
\sum_{k=0}^{\infty} G(k) z^{-k} & =V_{a}\left(z I_{r}-A_{r}\right)^{-1} U_{a}+V_{b} U_{b} \\
& =V_{a} \sum_{k=1}^{\infty} A_{r}^{k-1} z^{-k} U_{a}+V_{b} U_{b} .(14 \mathrm{a})
\end{aligned}
$$

Therefore, we have

$$
G(k)= \begin{cases}V_{b} \cdot U_{b} & \text { for } k=0 \\ V_{a} A_{r}^{k-1} U_{a} & \text { for } k=1,2, \ldots, \infty .\end{cases}
$$

The matrix $T$ can be readily obtained from equation (14b). Since all eigenvalues of $A_{r}$ are within unit disk $D(0,1)$, we can find a similarity transformation matrix $S$ such that $A_{r}=S D_{r} S^{-1}$, where $D_{r}$ is a block diagonal Jordan matrix. For simplicity, assume here $A_{r} \in \mathbb{R}^{5 \times 5}$ and $D_{r}$ has the following form:

$$
D_{r}=\left[\begin{array}{ccccc}
\lambda_{1} & 0 & 0 & 0 & 0 \\
0 & \lambda_{2} & 1 & 0 & 0 \\
0 & 0 & \lambda_{2} & 1 & 0 \\
0 & 0 & 0 & \lambda_{2} & 1 \\
0 & 0 & 0 & 0 & \lambda_{2}
\end{array}\right],
$$

where $\lambda_{1}$ and $\lambda_{2}$ are eigenvalues of $A_{r}$ with $\left|\lambda_{1}\right|<1$ and $\left|\lambda_{2}\right|<1$. The following inequality is obvious:

$$
\begin{aligned}
T= & \sum_{k=0}^{\infty}|G(k)|_{m} \\
\leq & \left|V_{b} U_{b}\right|_{m}+\left|V_{a}\right|_{m} \sum_{k=1}^{\infty}\left|A_{r}^{k-1}\right|_{m}\left|U_{a}\right|_{m} \\
\leq & \left|V_{b} U_{b}\right|_{m}+\left|V_{a}\right|_{m}|S|_{m} \sum_{k=0}^{\infty}\left|D_{r}^{k}\right|_{m} \\
& \times\left|S^{-1}\right|_{m} \mid U_{\left.a\right|_{m}} .
\end{aligned}
$$

Since $\left|\lambda_{i}\right|<1$ for $i=1,2$, it is not difficult to verify

$$
\begin{aligned}
& \sum_{k=1}^{\infty}\left|D_{r}^{k}\right|_{m} \leq \sum_{k=1}^{\infty}\left|D_{r}\right|_{m}^{k} \\
& =\left[\begin{array}{ccccc}
p_{1}\left|\lambda_{1}\right| & 0 & 0 & 0 & 0 \\
0 & p_{2}\left|\lambda_{2}\right| & p_{2}^{2} & p_{2}^{3} & p_{2}^{4} \\
0 & 0 & p_{2}\left|\lambda_{2}\right| & p_{2}^{2} & p_{2}^{3} \\
0 & 0 & 0 & p_{2}\left|\lambda_{2}\right| & p_{2}^{2} \\
0 & 0 & 0 & 0 & p_{2}\left|\lambda_{2}\right|
\end{array}\right],
\end{aligned}
$$

where $p_{1}=\frac{1}{1-\left|\lambda_{1}\right|}$ and $p_{2}=\frac{1}{1-\left|\lambda_{2}\right|}$. Therefore, all entries of matrix $T$ must be finite if the pair $(E, A)$ is asymptotically stable and impulse-free.

The following two lemmas will be used to prove Theorem 1.

Lemma 5. (Lancaster and Tismenetsky, 1985). For any $n \times n$ matrix $Q$, if $\rho(Q)<1$ then $|I-Q| \neq 0$.

$\Delta \Delta \Delta$

Lemma 6. (Chen, 1984, Theorem 3-4). Let $M(z)$ be a square rational matrix and be decomposed uniquely as $M(z)=M_{p}(z)+M_{s p}(z)$, where $M_{p}(z)$ is a polynomial matrix and $M_{s p}(z)$ is a strictly proper rational matrix. Then $M^{-1}(z)$ is proper if and only if $M_{p}^{-1}(z)$ exists and is proper.

$\Delta \Delta \Delta$

Theorem 1. Let the pair $(E, A)$ be regular, asymptotically stable and impulse-free. Assume the perturbation $\Delta A$ is bounded by $|\Delta A|_{m} \leq q H$ for some $q>0$. The perturbed system (1) is still regular, asymptotically stable and impulse-free if the following inequality:

$$
q<\frac{1}{\rho(T H)}
$$

holds, where $T$ is defined in equation (11).

Proof. Since $|z E-A|$ is not identically zero (i.e. regular), by the determinant formula, we have

$$
\begin{aligned}
\mid z E-A & -\Delta A \mid \\
& =|z E-A| \cdot\left|I-(z E-A)^{-1} \Delta A\right| .
\end{aligned}
$$

If inequality (18) holds, from Lemma 4 , we have $\rho\left((z E-A)^{-1} \Delta A\right)<1$ for all $z$ with $|z| \geq 1$. According to Lemma 5 , the following inequality:

$$
\left|I-(z E-A)^{-1} \Delta A\right| \neq 0 \text { for all } z \text { with }|z| \geq 1
$$

holds. In view of equation (19), we have $|z E-A-\Delta A| \neq 0$ for all $z$ with $|z| \geq 1$. Next we 
show the impulse-free and regular properties. If inequality (18) is satisfied, by Lemma 3 , one can get

$$
\begin{aligned}
\rho(G(0) \Delta A) \leq \rho\left(q|G(0)|_{m} H\right) & \\
& \leq \rho(q T H)<1 .
\end{aligned}
$$

The nonsingularity of $I-G(0) \Delta A$ is ensured obviously from Lemma 5 . The matrix $(z E-$ $A)^{-1}$ can be expressed as $(z E-A)^{-1}=G(0)+$ $G_{s p}(z)$, where $G_{s p}(z)$ is the strictly proper part. We rewrite

$$
\begin{aligned}
& (z E-A-\Delta A)^{-1} \\
& =\left[I-G(0) \Delta A-G_{s p}(z) \Delta A\right]^{-1}(z E-A)^{-1} .
\end{aligned}
$$

By Lemma 6 , the nonsingularity of $I-G(0) \Delta A$ implies that $\left[I-G(0) \Delta A-G_{s p}(z) \Delta A\right]^{-1}$ is proper and so is $(z E-A-\Delta A)^{-1}$. Therefore, by Lemma 2 , the perturbed system is impulse-free. If $(z E-A-\Delta A)^{-1}$ is proper, the system's regularity is also guaranteed (i.e. $|z E-A-\Delta A|$ is not identically zero).

Q.E.D.

Remark 2. From the above statements, we know that if the perturbed system is asymptotically stable and impulse-free, then the system regularity can be ensured as well. If $E=I$, from equations (11) and (14), then $T=\sum_{k=1}^{\infty}\left|A^{k-1}\right|_{m}$. The condition in equation (19) then coincides with Chou's results. Thus his results might be viewed as a special case of ours. In Chou's paper, he has showed many advantages of his approach. All his advantages are preserved in our approach to dealing with singular systems with highly structured uncertainties.

Remark 3. Many features of continuous-time singular systems and discrete-time singular systems are essentially different. For instance, stability of discrete-time systems can exclude the possibility of introducing the dynamical infinite modes, but this is not always true for the continuous-time case. The detailed derivation for continuous-time systems can be found in Fang and Lee (1993).

Example 1. Consider the following system:

$$
\begin{aligned}
{\left[\begin{array}{lll}
1 & 0 & 0 \\
0 & 0 & 0 \\
0 & 1 & 0
\end{array}\right] x(k+1) } \\
=\left[\begin{array}{ccc}
0.5 & 0 & 0 \\
4 & 0 & -2 \\
0 & 0.2 & 0
\end{array}\right] x(k)+\Delta A x(k) .
\end{aligned}
$$

The pair $(E, A)$ is regular, asymptotically stable and impulse-free. By Weierstrass decomposition, it is easy to obtain

$$
\begin{gathered}
U=\left[\begin{array}{ccc}
1 & 0 & 0 \\
0 & 0 & 1 \\
0 & 0.5 & 0
\end{array}\right], \quad V=\left[\begin{array}{lll}
1 & 0 & 0 \\
0 & 1 & 0 \\
2 & 0 & 1
\end{array}\right], \\
A_{r}=\left[\begin{array}{cc}
0.5 & 0 \\
0 & 0.2
\end{array}\right],
\end{gathered}
$$

then we have

$$
\begin{gathered}
G(0)=\left[\begin{array}{ccc}
0 & 0 & 0 \\
0 & 0 & 0 \\
0 & 0.5 & 0
\end{array}\right], \\
G(k)=\left[\begin{array}{ccc}
0.5^{k-1} & 0 & 0 \\
0 & 0 & 0.2^{k-1} \\
2 \cdot 0.5^{k-1} & 0 & 0
\end{array}\right], \\
\end{gathered}
$$

and

$$
T=\left[\begin{array}{ccc}
2 & 0 & 0 \\
0 & 0 & 1.25 \\
4 & 0.5 & 0
\end{array}\right]
$$

The following shows the allowable upper bounds on $q$ for various cases of structured perturbations:

$$
\text { (a) } H=\left[\begin{array}{lll}
1 & 0 & 0 \\
0 & 0 & 0 \\
0 & 0 & 0
\end{array}\right], \frac{1}{\rho(T H)}=0.5 \text {; }
$$

(i.e. the perturbed singular system in this example remains regular, asymptotically stable and impulse-free if the perturbation is bounded by $\left.|\Delta A|_{m}<0.5 H\right)$
(b) $H=\left[\begin{array}{lll}0 & 0 & 0 \\ 0 & 0 & 0 \\ 0 & 0 & 1\end{array}\right], \frac{1}{\rho(T H)}=\infty$;
(c) $H=\left[\begin{array}{lll}0 & 0 & 0 \\ 0 & 0 & 0 \\ 0 & 1 & 0\end{array}\right], \frac{1}{\rho(T H)}=0.2$;
(d) $H=\left[\begin{array}{lll}1 & 0 & 0 \\ 0 & 1 & 0 \\ 0 & 0 & 1\end{array}\right], \frac{1}{\rho(T H)}=0.5$;
(e) $H=\left[\begin{array}{lll}1 & 0 & 0 \\ 1 & 0 & 1 \\ 0 & 1 & 0\end{array}\right], \quad \frac{1}{\rho(T H)}=0.5$;
(f) $H=\left[\begin{array}{lll}1 & 1 & 1 \\ 1 & 1 & 1 \\ 1 & 1 & 1\end{array}\right], \frac{1}{\rho(T H)}=0.129$

Note the upper bounds of perturbations found in cases (a)-(c) are exact with respect to their 
perturbation structures. An interesting case should be indicated as follows. In some cases, the perturbations do not affect the positions of eigenvalues (i.e. eigenvalue sensitivity to perturbations is zero) but can destroy the regularity. Assume the perturbation is $\Delta A=\epsilon \cdot H_{1}$, where

$$
H_{1}=\left[\begin{array}{lll}
0 & 0 & 0 \\
0 & 0 & 1 \\
0 & 0 & 0
\end{array}\right] .
$$

It is easy to check

$$
|z E-A-\Delta A|=(2+\epsilon)(z-0.5)(z-0.2) \text {. }
$$

The finite eigenvalues of nominal system, $\lambda_{1,2}=0.5,0.2$, are not disturbed by the uncertainty $\epsilon \in(-2,2)$ for the perturbation structure $H_{1}$. However, the regularity will vanish suddenly when $\epsilon=-2$. Using Theorem 1, we have $1 / \rho\left(T H_{1}\right)=2$. This is the exact upper bound of perturbations for the remaining system to be regular.

Remark 4. Consider a continuous-time singular system $E \dot{x}(t)=A x(t)$. If one is only interested in whether the finite eigenvalues of the continuoustime system lie inside the unit disk $D(0,1)$. Theorem 1 can be directly applied to check this matter without any modification. Although this theorem is developed for discrete-time systems, the derivation still holds if the variable $z$ is replaced by $s$.

\subsection{Robust root-clustering in a specified disk}

The dynamic response of a linear timeinvariant system can be modified by means of placing the poles in predetermined locations. However, for systems with uncertain parameters, the exact placement of pole locations might be difficult to attain. Hence, the concept of pole placement within a specified region is a suitable and useful approach. Lately, many researchers have considered how to locate the closed-loop poles of a standard state-space system in a prescribed region to shape the dynamic response. However, the variation of pole locations due to parameter perturbations of the plant has not been discussed by them. Rachid (1989) and Fang (1993) have given some new methods to study the problem of root-clustering in a specified disk for standard state-space systems with linear time-invariant perturbations. The same problem for uncertain singular systems is still unsolved now.

In this section, we will apply the results derived in the previous section to solve the problem of robust root-clustering in a specified disk for uncertain singular systems. A sufficient condition is proposed to guarantee pole position robustness within a specified disk for singular systems with structured uncertainties. Under the allowable highly structured perturbation, both stability robustness and certain performance robustness can thus be ensured. Consider two singular systems.

and

$$
\Sigma_{1}: E x(k+1)=A x(k)+\Delta A x(k)
$$

$$
\Sigma_{2}: E x(k+1)=\hat{A} x(k)+\Delta \hat{A} x(k),
$$

where $\hat{A} \in \mathbb{R}^{n \times n}$ and $\Delta \hat{A}$ denotes the associated structured perturbation.

Definition 2. For any two $n \times n$ real constant matrices $X$ and $Y$, we say that all finite eigenvalues of the pair $(X, Y)$ lie inside disk $D(-h, f)$ if $(z X-Y)^{-1}$ is proper and all roots of $|z X-Y|=0$ are within the disk $D(-h, f)$.

Lemma 7. Assume $A$ and $\hat{A}$ are related by

$$
f \hat{A}=A+h E \text {. }
$$

Then, all finite eigenvalues of the pair $(E, A)$ lie inside disk $D(-h, f)$ if and only if all finite eigenvalues of the pair $(E, \hat{A})$ lie inside the unit disk $D(0,1)$.

Proof. This fact can be easily verified from the following identity:

$$
f(\lambda E-\hat{A})=z E-A,
$$

where $\lambda=(1 / f)(z+h)$.

Q.E.D.

Remark 5. In comparison with the nominal parts of systems $\Sigma_{1}$ and $\Sigma_{2}$, all finite eigenvalues of the pair $(E, A)$ lie inside disk $D(-h, f)$ if and only if the pair $(E, \hat{A})$ is asymptotically stable and impulse-free.

Assume all finite eigenvalues of the pair $(E, \hat{A})$ are located within disk $D(0,1)$ and the perturbation $\Delta \hat{A}$ can be bounded by

$$
|\Delta \hat{A}|_{m} \leq q \hat{H},
$$

where $q$ is a positive real number and $\hat{H}$ a nonnegative matrix. By the results of Theorem 1 , we can readily obtain that all finite eigenvalues of the perturbed system $\Sigma_{2}$ lie inside disk $D(0,1)$ (i.e. asymptotically stable and impulse-free) if the following inequality:

$$
q<\frac{1}{\rho(\hat{T} \hat{H})}
$$


is satisfied, where

$$
\begin{gathered}
\hat{T} \equiv \sum_{k=0}^{\infty}|\hat{G}(k)|_{m}, \\
(z E-\hat{A})^{-1}=\sum_{k=0}^{\infty} \hat{G}(k) z^{-k}
\end{gathered}
$$

and

$$
\hat{A} \equiv \frac{1}{f}(A+h E) .
$$

The robust root-clustering theorem is stated as follows.

Theorem 2. Assume all finite eigenvalues of the pair $(E, A)$ lie inside the disk $D(-h, f)$ and the perturbation $\triangle A$ is bounded by

$$
|\Delta A|_{m} \leq q H \text { for some } q>0 .
$$

Then all finite eigenvalues of the perturbed system $\Sigma_{1}$ still lie inside disk $D(-h, f)$ if the following inequality:

$$
q<\frac{f}{\rho(\hat{T} H)}
$$

holds, where $\hat{T}$ is defined in equation (28).

Proof. Let

$$
\Delta A=f \Delta \hat{A} .
$$

Comparing equations (31) and (26), we have $H=f \hat{H}$. Then the inequalities in (32) and the inequalities in (27) are equivalent. If all finite eigenvalues of the pair $(E, A)$ lie in disk $D(-h, f)$, then from Lemma 7 all finite eigenvalues of the pair $(E, \hat{A})$ are located within the unit circle $D(0,1)$. Define $\Phi$ and $\Phi$ as

$$
\Phi \equiv A+\Delta A=A+f \Delta \hat{A}
$$

and

$$
\hat{\Phi} \equiv \hat{A}+\Delta \hat{A} .
$$

From Theorem 1, if inequality (32) [or inequality (27)] is satisfied, we can say that all finite eigenvalues of the pair $(E, \Phi)$ are located inside disk $D(0,1)$. Since $\Phi$ and $\hat{\Phi}$ are related by

$$
f \hat{\Phi}=\Phi+h E
$$

it is easy to check by Lemma 7 that all finite eigenvalues of the pair $(E, \Phi)$ lie in disk $D(-h, f)$. Thus all finite eigenvalues of the perturbed system $\Sigma_{1}$ are still located in disk $D(-h, f)$, under the perturbation $\Delta A$. Q.E.D.

Remark 6. The techniques of Theorem 2 with equations (27)-(30) can also be applied to solve the problems of root-clustering robustness inside a disk centred on the negative real axis for continuous-time systems without any modification.
Example 2.

$$
\begin{aligned}
{\left[\begin{array}{lll}
1 & 0 & 0 \\
0 & 0 & 0 \\
0 & 1 & 0
\end{array}\right] x(k+1) } & \\
= & {\left[\begin{array}{ccc}
0 & 0.25 & 0 \\
1 & 0 & -0.5 \\
-0.75 & -1 & 0
\end{array}\right] x(k)+\Delta A x(k) . }
\end{aligned}
$$

The pair $(E, A)$ is impulse-free and its finite eigenvalues are $\lambda_{1}=-0.25$ and $\lambda_{2}=-0.75 \epsilon$ $D(-0.5,0.5)$. If $\hat{A}=(1 / 0.5)(A+0.5 E)$, it is easy to check that the finite eigenvalues of the pair $(E, \hat{A})$ are $\lambda_{1}=\frac{1}{2}$ and $\lambda_{2}=-\frac{1}{2} \in D(0,1)$. Using equation (28), we obtain

$$
\hat{T} \approx\left[\begin{array}{ccc}
2.6667 & 0 & 0.6667 \\
2 & 0 & 2.6667 \\
5.3333 & 1 & 1.3333
\end{array}\right] .
$$

If the structured perturbation information is

$$
H=\left[\begin{array}{lll}
1 & 0 & 0 \\
0 & 1 & 0 \\
0 & 0 & 1
\end{array}\right]
$$

then $0.5 / \rho(\hat{T} H)=0.1161$. Hence if $|\Delta A|_{m}<$ $0.1161 H$, not only is the perturbed system regular and impulse-free but all finite eigenvalues of the perturbed system are still located within $D(-0.5,0.5)$. If the perturbation structure is changed to

$$
H=\left[\begin{array}{lll}
1 & 1 & 1 \\
1 & 1 & 1 \\
1 & 1 & 1
\end{array}\right],
$$

then $0.5 / \rho(\hat{T} H)=0.0319$.

\subsection{Robust control design}

Based on the results derived in Section 4.1 and 4.2 , we now propose a simple method to synthesize a controller for robust pole assignment of perturbed singular systems in a specified region. Let us consider a perturbed singular system

$$
E x(k+1)=(A+\Delta A) x(k)+(B+\Delta B) u(k)
$$

where $B \in \mathbb{R}^{n \times m}$. We only assume the triple $(E, A, B)$ is strongly controllable (Verghese $e t$ al., 1981). In equation (37), $\Delta A$ and $\Delta B$ are linear time-invariant perturbations which can be bounded by

$$
|\Delta A|_{m} \leq q_{1} H_{1} \text { and }|\Delta B|_{m} \leq q_{2} H_{2},
$$

where $q_{i}, i=1,2$ are both positive real numbers, and $H_{i}, i=1,2$ are two nonnegative constant matrices. Here we assume the perturbation 
bounds $q_{1}$ and $q_{2}$ and the structured perturbation information $H_{1}$ and $H_{2}$ are known in advance.

Let the static state feedback control be

$$
u(k)=F x(k)
$$

Then the nominal closed-loop system is

$$
E x(k+1)=(A+B F) x(k)
$$

and the perturbed closed-loop system

$$
E x(k+1)=(A+B F) x(k)+(\Delta A+\Delta B F) x(k) .
$$

Therefore, the design problem to be considered is to determine the feedback gain $F$ in (39), such that all finite poles of the nominal closed-loop system (40) lie inside a specified disk, and at the same time all finite poles of the perturbed closed-loop system (41) are also located within the same disk. Suppose the specified region for robust root-clustering is the interior of disk $D(-h, f)$, then employing Theorem 2 , we have the following result.

Theorem 3. All finite closed-loop poles of the perturbed system (41) will be located within the disk $D(-h, f)$, if $\Delta A$ and $\Delta B$ are bounded by (38), all finite eigenvalues of the pair $(E, A+$ $B F)$ lie inside a disk $D(-h, f)$, and the following inequality:

$$
q_{1}<\frac{f}{\rho(\bar{T} \bar{H})}
$$

is satisfied, where

$$
\bar{T}=\sum_{k=0}^{\infty}|\bar{G}(k)|_{m}
$$

and

$$
\bar{H}=H_{1}+\frac{q_{2}}{q_{1}} H_{2}|F|_{m} .
$$

In equation (43), $\bar{G}(k)$ is obtained by

$$
(z E-\bar{A})^{-1}=\sum_{k=0}^{\infty} \bar{G}(k) z^{-k},
$$

where

$$
\bar{A}=\frac{1}{f}(A+B F+h E)
$$

Proof. From equation (38) and Lemma 2, we have

$$
\begin{aligned}
|\Delta A+\Delta B F|_{m} & \leq|\Delta A|_{m}+|\Delta B|_{m}|F|_{m} \\
& \leq q_{1} H_{1}+q_{2} H_{2}|F|_{m} \\
& =q_{1} \bar{H} .
\end{aligned}
$$

Therefore, according to Theorem 2, we have the stated results.

Q.E.D.
Now the following design algorithm, for a given set of perturbations, can be used to select the control gain $F$ for which the system is pole-assignment robust.

Step 1 . Specify the finite closed-loop eigenvalues $\lambda_{i}(i=1,2, \ldots, r)$ in the specified disk $D(-h, f)$.

Step 2. Use any pole-assignment technique of singular systems to design $F$ (Wang et al., 1987; Fahmy and O'Reilley, 1989; Fang and Chang, 1990; Kucera and Zagalak, 1991; Paraskevopoulos and Koumboulis, 1991), such that the nominal closed-loop system (40) has the specified $\lambda_{i}$.

Step 3. Based on highly structured information (38) and on the designed $F$, check if the robust pole-assignment condition given in (42) is satisfied. If so, the design of robust controller for robust pole-assignment is finished. If not, since the different eigenstructure can help in satisfying the condition in (42), we shrink the nominal closed-loop eigenvalues closer to the centre of the specified disk and then go back to Step 2.

Remark 7. The inequality (42) indicates that the smaller the value $\rho(\bar{T} \bar{H})$, the more robust the controller becomes. It has been shown that (Lancaster and Tismenetsky, 1985)

$$
\rho(\bar{T} \bar{H}) \leq\|\bar{T} \bar{H}\| \leq\|\bar{T}\|\|\bar{H}\|,
$$

where $\|\cdot\|$ denotes any matrix norm. In some cases, one may want to choose the gain $F$ to assign the nominal closed-loop poles to some predetermined positions in the specified disk. In MIMO systems, the gains $F$ are not unique. Since the matrices $\bar{T}$ and $\bar{H}$ are functions of $F$, the gain $F$ may be selected so that the product of $\|\bar{T}\|$ and $\|\bar{H}\|$ is as small as possible for obtaining robust stability. In general, if a gain $F$ is chosen to make $\|\bar{T}\|$ decrease (increase), it will increase (decrease) $\|\bar{H}\|$. The next example will display this interesting observation. The exact relation between the choice of $F$ and these two matrix norms is still not clear.

Example 3. Consider a perturbed discrete-time singular system

$$
\begin{aligned}
{\left[\begin{array}{lll}
1 & 0 & 0 \\
0 & 0 & 0 \\
0 & 1 & 0
\end{array}\right] x(k+1) } & =\left[\begin{array}{ccc}
-3.2 & -1.6 & 0 \\
0 & 0 & -1 \\
0.8 & 2.4 & 0
\end{array}\right] x(k) \\
+ & {\left[\begin{array}{ll}
1 & 0 \\
1 & 0 \\
0 & 1
\end{array}\right] u(k)+\Delta A x(k)+\Delta B u(k) }
\end{aligned}
$$


which has finite eigenvalues at $\lambda_{1,2}=2.1612$, -2.1612 . Assume $\Delta A$ and $\Delta B$ are bounded by

$$
|\Delta A|_{m} \leq q_{1} H_{1}=0.2 \cdot\left[\begin{array}{lll}
0 & 0 & 0 \\
0 & 0 & 1 \\
0 & 1 & 0
\end{array}\right]
$$

and

$$
|\Delta B|_{m} \leq q_{2} H_{2}=0.01 \cdot\left[\begin{array}{ll}
1 & 0 \\
0 & 0 \\
0 & 1
\end{array}\right] .
$$

Select a feedback gain

$$
F=\left[\begin{array}{ccc}
3.2 & 2.4 & 0 \\
-1.2 & -2 & 0
\end{array}\right]
$$

then the nominal closed-loop system has finite eigenvalues at $\lambda_{1}=0.2+\mathrm{j} 0.5292$ and $\lambda_{2}=0.2-$ j0.5292 $\in D(0,0.8)$. From equation (43), we have

$$
\bar{T} \approx\left[\begin{array}{ccc}
2.3191 & 0 & 2.6383 \\
1.3191 & 0 & 2.6383 \\
9.6662 & 0.8 & 12.9324
\end{array}\right] .
$$

Since

$$
\bar{H}=H_{1}+\frac{q_{2}}{q_{1}} H_{2}|F|_{m}=\left[\begin{array}{ccc}
0.16 & 0.12 & 0 \\
0 & 0 & 1 \\
0.06 & 1.1 & 0
\end{array}\right]
$$

and

$$
q_{1}=0.2<\frac{0.8}{\rho(\bar{T} \bar{H})}=0.2311
$$

the feedback closed-loop system is impulse-free and all its finite eigenvalues would stay inside $D(0,0.8)$ under the perturbations for such feedback gain.

If the feedback gain is selected as

$$
F=\left[\begin{array}{ccc}
3.1 & 0 & 0 \\
-0.8 & -2.4 & 0
\end{array}\right]
$$

the finite eigenvalues of the nominal closed-loop system are $\lambda_{1}=0.1$ and $\lambda_{2}=0$ which are closer to the centre of $D(0,0.8)$. Then we compute the corresponding $\bar{T}$ and $\bar{H}$

$$
\begin{gathered}
\bar{T} \approx\left[\begin{array}{ccc}
1.1429 & 0 & 2.2857 \\
0 & 0 & 1 \\
3.5429 & 0.8 & 7.0857
\end{array}\right], \\
\bar{H}=\left[\begin{array}{ccc}
0.155 & 0 & 0 \\
0 & 0 & 1 \\
0.04 & 1.12 & 0
\end{array}\right]
\end{gathered}
$$

and obtain

$$
q_{1}=0.2<\frac{0.8}{\rho(\bar{T} \bar{H})}=0.6520 .
$$

It tells us that the feedback closed-loop system can now tolerate perturbations larger than the former feedback system can. It is true for most systems.
In MIMO systems, many different feedback gains could be selected for the same poleassignment locations. The following shows that the selection of feedback gains will affect the permissible perturbations. For assigning both eigenvalues to the origin, let

$F_{1}=\left[\begin{array}{ccc}3.2 & 1.6 & 0 \\ -0.8 & -2.4 & 0\end{array}\right]$ and $F_{2}=\left[\begin{array}{ccc}2.2 & 0.6 & 0 \\ 0.2 & -1.4 & 0\end{array}\right]$

be applied, respectively. Then using Theorem 3 , we find their corresponding $\bar{T}$ and $\bar{H}$

$\begin{array}{ll}\bar{T}_{1} \approx\left[\begin{array}{ccc}1 & 0 & 0 \\ 0 & 0 & 1 \\ 3.2 & 0.8 & 1.6\end{array}\right], & \bar{T}_{2} \approx\left[\begin{array}{ccc}2.25 & 0 & 1.25 \\ 1.25 & 0 & 2.25 \\ 4.2 & 0.8 & 2.6\end{array}\right], \\ \bar{H}_{1}=\left[\begin{array}{ccc}0.16 & 0.08 & 0 \\ 0 & 0 & 1 \\ 0.04 & 1.12 & 0\end{array}\right], \quad \bar{H}_{2}=\left[\begin{array}{ccc}0.11 & 0.03 & 0 \\ 0 & 0 & 1 \\ 0.01 & 1.07 & 0\end{array}\right]\end{array}$

and upper bounds of associated $q_{1}$

$$
\frac{0.8}{\rho\left(\bar{T}_{1} \bar{H}_{1}\right)}=0.7122 \text { and } \frac{0.8}{\rho\left(\bar{T}_{2} \bar{H}_{2}\right)}=0.3314 \text {. }
$$

Hence the resultant permissible ranges of $q_{1}$ are quite different. Note that $\left\|\bar{T}_{1}\right\|_{\infty}=5.6, \mid \bar{T}_{2} \|_{\infty}=$ 7.6, $\left\|\bar{H}_{1}\right\|_{\infty}=1.16$, and $\left\|\bar{H}_{2}\right\|_{\infty}=1.08$.

Remark 8. It is noted that we may have to try many initial estimates of the nominal closed-loop eigenvalues before we find the desired feedback gain matrix for robust pole-assignment.

\section{CONCLUSIONS}

The robust control analysis and design for discrete-time singular systems are studied in this paper. A sufficient condition for simultaneously checking stability robustness, regularity robustness, and impulse-free robustness of singular systems, under highly structured perturbation, is provided. Only simple computations are needed in this approach. Based on the criteria developed, we also provide a qualitative algorithm to design a robust controller for assigning poles inside a specified region. In the literature, little effort has been devoted to dealing with the problem of robust control analysis and design for singular systems. This paper may be viewed as complementary in this field. We also believe that the same problem can be solved by using the Lyapunov equation, as studied for standard state-space systems. By the proposed criterion, the computed upper bounds of perturbations may be exact for certain structures, but in some cases it may be too conservative. Therefore, to improve the 
criterion for obtaining a less conservative bound is also under investigation.

Acknowledgements-The authors would like to thank the anonymous reviewers for their valuable comments and suggestions. The first author is grateful to Professor Jer-Guang Shieh in the Department of Electrical Engineering at National Sun Yat-Sen University, Taiwan for his encouragement.

\section{REFERENCES}

Bender, D. J. and A. J. Laub (1987). The linear-quadratic optimal regulator for descriptor systems. IEEE Trans. Automat. Control, 32, 672-687.

Chen, C. T. (1984). Linear System Theory and Design. Holt Rinehart and Winston, NY.

Chou, J. H. (1990). Stability robustness of linear state space models with structured perturbations. Systems \& Control Letters, 15, 207-210.

Cobb, J. D. (1984). Controllability observability, and duality in singular systems. IEEE Trans. Automat. Control, 29, 1076-1082.

Dai, L. (1989). Singular Control Systems--Lecture Notes in Control and Information Sciences. Springer-Verlag, Berlin.

Fahmy, M. M. and J. O'Reilley (1989). Parametric eigenstructure assignment for continuous-time descriptor systems. Int. J. Control, 49, 129-143.

Fang, C. H. (1993). Pole-assignment robustness in a ring for structured perturbation systems. In Proc. of 1993 IEEE Symp. on Circuits and Systems, pp. 2510-2513.

Fang, C. H. and F. R. Chang (1990). Deadbeat control in singular systems and its applications. Control-Theory and Advanced Technology (C-TAT), 6, 383-393.

Fang, C. H. and F. R. Chang (1991). A new application of infinite eigenvalue assignment in generalized state-space systems. In Proc. of 30th IEEE CDC, 1, pp. 921-922.

Fang, C. H. and F. R. Chang (1992). A simple approach to solving the Diophantine equation. IEEE Trans. Automat. Control, 1, 152-155.

Fang, C. H. and L. Lee (1993). Regional pole-assignment robustness for uncertain continuous-time implicit systems. In Proc. of 32nd IEEE CDC, 4, pp. 3319-3324.

$\mathrm{He}$, J. and G. Ji (1992). Practical stability of large scale dynamic systems with impulsive solutions. IFAC/IFCRS/IMACS Symp. Large Scale Systems Theory and Appl., 585-591.

Juang, Y. T. (1991). Robust stability and robust pole assignment of linear systems with structured uncertainty. IEEE Trans. Automat. Control, 36, 635-637.

Kucera, V. and P. Zagalak (1988). Fundamental theorems of state feedback for singular systems. Automatica, 24, $653-658$

Kucera, V. and P. Zagalak (1991). Eigenstructure assignment by PD state feedback in linear systems. In Proc. of 30 th IEEE CDC, pp. 1284-1286.

Lancaster, P. and M. Tismenetsky (1985). The Theory of Matrices. 2nd edition, Academic Press, NY.

Lewis, F. L. (1986). A survey of linear singular systems. $J$. Circuit, Syst., Signal Processing, 5, 3-36.

Luenberger, D. G. (1977). Dynamic equations in descriptor form. IEEE Trans. Automat. Control, 22, 312-321.

Mertzios, B. G. (1984). On the sensitivity analysis of linear time-invariant singular systems. IEEE Trans. Circuits and Systems, 31, 978-982.

Nichols, N. K. (1986). Robust control system design for generalized state-space systems. In Proc. of 25th IEEE $C D C$, pp. 538-540.

Paraskevopoulos, P. N. and F. N. Koumboulis (1991). Decoupling and pole assignment in generalized state space systems. IEE Pt.D, 138, 547-560.

Qiu, L. and E. J. Davison (1992). The stability robustness of generalized eigenvalues. IEEE Trans. Automat. Control 37, 886-891.

Rachid, A. (1989). Robustness of discrete systems under structured uncertainties. Int. J. Control, 50, 1563-1566.

Sobel, K. M., S. S. Banda and H. H. Yeh (1989). Robust control for linear systems with structured state space uncertainty. Int. J. Control, 50, 1991-2004.

Syrmos, V. L. and F. L. Lewis (1991). Robust eigenvalue assignment in generalized systems. In Proc. of 30th IEEE $C D C$, pp. 1433-1434.

Shayman, M. A. (1987). Homogeneous indices, feedback invariants, and control structure theorem for generalized linear systems. SIAM J Contr. Opt., 32, 42-50.

Tesi, A. and A. Vicino (1990). Robust stability of state-space models with structured uncertainties. IEEE Trans. Automat. Control, 35, 191-195.

Verghese, G. C., B. C. Levy and T. Kailath (1981). A generalized state-space for singular systems. IEEE Trans. Automat. Control, 26, 811-831.

Wang, Y. Y., S. J. Shi and Z. J. Zhang (1987). Pole placement and compensator design of generalized systems. Systems \& Control Letters, 8, 205-209.

Yedavalli, R. K. (1993). A Kernecker based theory for robust root clustering of linear state-space models with real parameter uncertainty. In Proc. of 1993 ACC, pp. 2755-2759.

Zhou, Z., M. A. Shayman and T. J. Tarn (1987). Singular systems: a new approach in the time domain. IEEE Trans. Automat. Control, 32, 42-50. 